\title{
State of health estimation of lithium-ion battery packs in electric vehicles based on genetic resampling Particle Filter
}

\author{
Jun $\mathrm{Bi}^{\mathrm{a}, \mathrm{b}}$, Ting Zhang ${ }^{\mathrm{a}, \mathrm{b}}$, Haiyang $\mathrm{Yu}^{\mathrm{c}}$, Yanqiong Kang ${ }^{\mathrm{a}}$ \\ ${ }^{a}$ School of Traffic and Transportation, Beijing Jiaotong University, Beijing 100044, China \\ ${ }^{b}$ MOE Key Laboratory for Urban Transportation Complex Systems Theory and Technology \\ ${ }^{\mathrm{c}}$ School of Transportation Science and Engineering, Beijing Key Laboratory for Cooperative Vehicle \\ Infrastructure, Systems, and Safety Control, Beihang University, Beijing 100191, China
}

\begin{abstract}
:
Power battery packs are the energy source of battery electric vehicles (BEVs). A precise battery state of health $(\mathrm{SOH})$ estimation is crucial to ensure the security and stability of the operation of BEVs. Hence, a battery pack model is established in this study. Given that a battery pack is a complex and nonlinear system, the proposed battery pack model is simplified to reduce computational complexity, which usually produces non-Gaussian noise. Considering that the genetic resampling particle filter (GPF) performs efficiently in solving nonlinear and non-Gaussian problems, we propose a new GPF-based method for battery SOH estimation. First, a second-order equivalent circuit model of Resistance-Capacitance (RC) circuit for the battery pack is developed. The unknown parameters are identified using the recursive least squares with forgetting factor. Second, a state-space model of the GPF is developed on the basis of the equivalent circuit model. Finally, we investigate the estimation performance of the proposed model by conducting a case study using the real data collected from operating electric taxis in Beijing. Estimation results show that the proposed GPF model outperforms the particle filter method in the SOH estimation problem.
\end{abstract}

KEYWORDS: state of health; battery pack model; least-squares estimation; particle filter algorithm; genetic algorithm.

\section{Introduction}

As a result of environment deterioration and energy shortage, battery electric vehicles (BEVs) that use electric energy instead of fossil fuels are playing a crucial role in the automotive industry. Thus, the Chinese government has been implementing programs for the research, demonstration, and deployment of BEVs for over 20 years.

Li-ion batteries exhibit high energy density, minimal weight, long cycle life, high power capability, and low environmental impact [1-3].As such, these batteries have become the preferred energy storage equipment for BEVs. Multitudinous cells are

\footnotetext{
${ }^{*}$ Corresponding author.

School of Transportation Science and Engineering, Beijing Key Laboratory for Cooperative Vehicle Infrastructure, Systems, and Safety Control, Beihang University, Beijing, China, 100191.
}

Tel.: +86 18612452770. E-mail address: hyyu@buaa.edu.cn (Haiyang Yu) 
connected in series and parallel to a battery pack because the limited voltage and capacity of a cell cannot meet practical requirements [4].The life of a battery pack, which is also called state of health $(\mathrm{SOH})$, directly reflects the aging condition of a battery pack and its ability to deliver the specified performance in comparison with a fresh one. Thus, $\mathrm{SOH}$ is a crucial factor to realize the timely maintenance and replacement of battery systems. As BEVs derive all power from battery packs and $\mathrm{SOH}$ affects the dynamic behavior of electric vehicles (e.g., speed and mileage), a research on the $\mathrm{SOH}$ of battery packs is critical to guarantee the reliable operation of BEVs. However, ensuring the accuracy of battery pack SOH estimation under working conditions remains a challenge.

In practice, $\mathrm{SOH}$ cannot be measured directly. Previous studies always used other indicators to calculate $\mathrm{SOH}$. At present, three indexes are mainly used for battery $\mathrm{SOH}$ estimation: behavior of charge and discharge and cycle numbers, battery capacity degradation, and internal resistance change. The first index, which is based on the analysis of charge and discharge performance, is the initial method [5-7] that usually requires numerous current charge and discharge tests. The experimental equipment is also expensive. As such, this approach may entail a time-consuming and costly testing of cycling life. Moreover, excessive discharge affects battery life [8,9]. Therefore, this method is usually used for laboratory research. The second index, which is based on capacity change, is most commonly adopted for $\mathrm{SOH}$ estimating [10-13]. Battery capacity cannot be directly measured during operation; thus, previous works used many algorithms to calculate capacity degradation to estimate $\mathrm{SOH}$. These algorithms include Support vector machine (SVM) [11,14], Extended Kalman filter (EKF)[15,16], Gaussian process regression[17], and Particle filter(PF)[18].

The relationship between $\mathrm{SOH}$ and internal resistance is strong, and the latter can dynamically track the former. [19,20]. To timely estimate $\mathrm{SOH}$ online, many researches adopt resistance as an index to represent $\mathrm{SOH}$. In addition, resistance cannot be acquired directly; it is calculated on the basis of other algorithms. A battery model should be established to compute resistance. The model parameters are used for $\mathrm{SOH}$ estimation in [21,22].SVM is also an effective method for battery $\mathrm{SOH}$ estimation based on the computation of resistance [23,24]. However SVM is a statistical approach based on a large amount of data, and it considers the battery system as a black box rather than a practical circuit model. The black-box model cannot describe the internal mechanism of the mathematical and physical structure. Thus it is not suitable for an in-depth investigation on the mechanism of the effect indicators of $\mathrm{SOH}$. Eliminating all non-stationarity in a dataset may not be possible even through repeated difference. Therefore, SVM may not accurately estimate the $\mathrm{SOH}$ in practice. KF is a powerful intelligent method to accurately estimate the state of any dynamic system [25].Unlike SVM, KF requires a battery state-space model. In many studies, KF was improved to identify ohmic resistance for $\mathrm{SOH}$ estimation; such improvement produced the EKF [26,27], Unscented Kalman Filtering (UKF) [28], Dual Extended Kalman filter Kalman filter (DEKF) [29,30]. KF is a promising 
estimation method for linear systems with Gaussian noise. However, handling a complex nonlinear system (e.g., BEVs) remains difficult for KF-based methods because the estimation results may disclose large errors or even lead to divergence. Unlike the KF algorithm, which can be used for linear systems with Gaussian noise, the PF algorithm uses the approximate sampling of different probability distributions and is equivalent to a posterior probability density function based on Monte Carlo theory, which is applicable to complex and nonlinear systems [31].The PF algorithm has been successfully and extensively used to dynamically estimate and track targets because of its capability of achieving approximately optimal estimation.

The BEV battery system is a strongly nonlinear system consisting of multitudinous Li-ion single cells connected in series and parallel or series/parallel. Inconsistencies between the cells lead to varying work conditions and seriously affect the life of battery packs [32].SOH estimation requires the evaluation of battery behavior to develop a high fidelity equivalent circuit model at the battery pack level from the perspective of the system $[33,34]$.A previous work regarded the battery pack model as a linear system, and research on cells remains impractical. The number of $\mathrm{RC}$ networks determines the resolution of the dynamic responses of the battery model [35].Given that the BEV battery pack is a complex and nonlinear system, the battery pack model always consists of $\mathrm{N}$ sets of RC networks to meet requirements. However, additional RC networks can increase calculation. Conventionally, computational complexity in actual engineering is reduced with the simplification of the battery pack model, although doing so usually produces non-Gaussian noise. In conclusion, $\mathrm{SOH}$ estimation involves solving nonlinear and non-Gaussian problems, and such process is the strength of the PF algorithm [36].Therefore, using the PF algorithm for the $\mathrm{SOH}$ estimation of BEV battery packs is logical. However, the degradation problem in the PF algorithm significantly affects estimation accuracy. To solve this problem and improve the estimation accuracy, we adopt the genetic algorithm in the resampling of PF [37-39], which is called the genetic resampling particle filter (GPF). Previous works showed that the GPF performs better than the PF. Thus, the GPF may be suitable for the SOH estimation of BEV battery packs.

Battery pack resistance during charging is used for battery $\mathrm{SOH}$ estimation in this study. First, a BEV battery equivalent circuit model is established, and the corresponding parameters are identified. A resistance state equation and an observational equation are modeled on the basis of the battery equivalent circuit model. Then, the GPF is introduced to dynamically track resistance and estimate $\mathrm{SOH}$ in reference to real operation data from eight electric taxis in Beijing. The organization of the study is as follows. The BEV battery equivalent circuit is modeled and corresponding parameters are identified via the recursive least squares estimation with forgetting factor in Section 2. The standard PF and GPF for the SOH estimation problem are presented in Section 3. To investigate the prediction performance of the proposed method, a comparative study is conducted in Section 4. Finally, conclusions are drawn in Section 5. 


\section{Equivalent circuit model for lithium-ion battery}

\subsection{Lithium-ion battery pack model}

The battery pack model should include the dynamic and static characteristics of battery packs to reflect the dynamic changing process of battery $\mathrm{SOH}$. Massimo Ceraolo proposed a dynamic RC model that can describe the electrode reaction, energy distribution, and ohmic effect in batteries [40].This model is extensively used in the industry. In general, a higher-order RC circuit model can better reflect the battery behavior precisely. However, a high order usually equates to a complex computation. Therefore, to make a trade-off between accuracy and computation complexity, this study develops an $\mathrm{SOH}$ estimation model on the basis of a second-order RC Massimo Ceraolo equivalent circuit model.

The second-order RC equivalent circuit model is shown in Fig. 2-1, which describes the dynamic characteristics of a battery pack. In the model, $V$ represents the battery terminal voltage. $V_{o c}$ is the open-circuit voltage, which has a nonlinear relationship with state of charge (SOC). $R_{0}$ is the internal ohmic resistance, which is the indicator of the battery $\mathrm{SOH}$. The sum of $R_{1}$ and $R_{2}$ represents the polarization resistance.

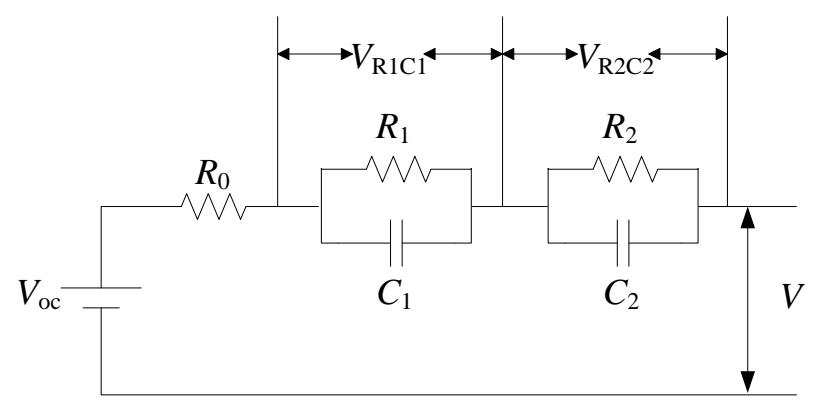

Fig. 1. Second-order RC model of battery pack

According to the second-order RC equivalent circuit model, the $V_{o c}$ can be described as:

$$
V_{o c}=\left(\frac{R_{1}}{R_{1} C_{1} s+1}+\frac{R_{2}}{R_{2} C_{2} s+1}+R_{0}\right) I+V
$$

We define $\tau_{1}=R_{1} C_{1}, \tau_{2}=R_{2} C_{2}, a=\tau_{1} \tau_{2}, \quad b=\tau_{1}+\tau_{2} ， \quad c=R_{0}+R_{1}+R_{2}$ ， $d=R_{1} \tau_{2}+R_{2} \tau_{1}+R_{0}\left(\tau_{1}+\tau_{2}\right)$, and substitute them into Eq. (2-1) to obtain Eq. (2-2).

$$
a V_{o c} s^{2}+b V_{o c} s+V_{o c}=a R_{0} I s^{2}+d I s+c I+a V s^{2}+b V s+V
$$

Eq. (2-3) is the differential equation of the battery pack model.

$$
\begin{gathered}
V_{o c}(k)-V(k)=k_{1}\left[V(k-1)-V_{o c}(k-1)\right]+k_{2}\left[V(k-2)-V_{o c}(k-2)\right]+ \\
k_{3} I(k)+k_{4} I(k-1)+k_{5} I(k-2)
\end{gathered}
$$

where $\mathrm{T}$ is the sampling period, and 
$k_{1}=\frac{-b T-2 a}{T^{2}+b T+a}, \quad k_{2}=\frac{a}{T^{2}+b T+a}, \quad k_{3}=\frac{c T^{2}+d T+a R_{0}}{T^{2}+b T+a}$,

$k_{4}=\frac{-d T-2 a R_{0}}{T^{2}+b T+a} \quad k_{5}=\frac{a R_{0}}{T^{2}+b T+a}$

From the relationship between $\mathrm{a}, \mathrm{b}, \mathrm{c}$, and $\mathrm{d}$ and $\tau_{1}, \tau_{2}, R_{0}, R_{1}$, and $R_{2}$, we can derive Eq. (2-4) :

$$
\begin{gathered}
R_{0}=k_{5} / k_{2}, \quad R_{2}=\left(\tau_{2} c+R_{0} b-\tau_{2} R_{0}-d\right) /\left(\tau_{2}-\tau_{1}\right), \quad R_{1}=c-R_{2}-R_{0} \\
\tau_{1}=\min \left(\frac{b+\sqrt{b^{2}-4 a}}{2}, \frac{b-\sqrt{b^{2}-4 a}}{2}\right) \\
\tau_{2}=\max \left(\frac{b+\sqrt{b^{2}-4 a}}{2}, \frac{b-\sqrt{b^{2}-4 a}}{2}\right)
\end{gathered}
$$

On the basis of Eq. (2-3) and (2-4), the parameters $R_{0}, R_{1}, R_{2}, C_{1}$, and $C_{2}$ of the second-order equivalent circuit model can be calculated.

\subsection{Parameter identification}

Various methods can be used to identify the parameters of the battery model. The least squares method is widely used to estimate system parameters because of its simple principle and fast convergence [41]. The recursive least square method with forgetting factor [42] is based on the gradually improved least square method, which mainly overcomes the "data saturation" phenomenon emerging with the growth of data. Thus, the recursive least squares method with forgetting factor is adopted to identify the parameters of the battery model, which is based on the mass data of the actual operation of an electric vehicle.

If a system is described with a linear differential equation as

$$
y(k)=\varphi(k) \theta+\xi(k),
$$

where $\varphi(k)$ is the input vector and $\theta$ is the parameter vector, $y(k)$ is the system output, and $k$ is the sample time. We define $\hat{\theta}$ as the estimated parameter vector. Then, the output estimation is

$$
\hat{y}(k)=\varphi(k) \hat{\theta} .
$$

Thus, according to the BEV battery model,

$$
\begin{gathered}
\varphi(k)=\left[V(k-1)-V_{o c}(k-1), V(k-2)-V_{o c}(k-2), I(k), I(k-1), I(k-2)\right]^{T} \\
\hat{\theta}=\left[k_{1}, k_{2}, k_{3}, k_{4}, k_{5}\right] .
\end{gathered}
$$

According to Eq. (2-3), $\hat{y}(k)$ equals $V_{\text {oc }}(k) \_V(k)$.

The forgetting factor recursive least squares method is defined by 


$$
\left\{\begin{array}{l}
\hat{\theta}(k)=\hat{\theta}(k-1)+K(k)\left[y(k)-\varphi^{T}(k) \hat{\theta}(k-1)\right] \\
K(k)=\frac{P(k-1) \varphi(k)}{\lambda+\varphi^{T}(k) P(k-1) \varphi(k)} \\
P(k)=\frac{1}{\lambda}\left[I-K(k) \varphi^{T}(k)\right] P(k-1)
\end{array},\right.
$$

where $\lambda$ is the forgetting factor value, which must be a positive number. Usually, $\lambda$ is in $[0,1]$ and is not less than 0.9 . If the system is linear, then range of $\lambda$ is $[0.95$, 1].

The steps of parameter $\left(k_{1}, k_{2}, k_{3}, k_{4}, k_{5}\right)$ identification are simply described as follows:

Step1: Calculate the open-circuit voltage $V_{o c}$ of the charging process on the basis of $V_{o c}=A+B \xi$, where $B=[1: 1: n]$ and $\mathrm{A}$ is the initial terminal voltage. $\xi$ is a random number within a small interval that is used to express the slow change of $V_{o c}$ in charging. $n$ is the number of iterations that is set to 500 .

Step2: Set the initial value of the algorithm. Set $\hat{\theta}=(0,0,0,0,0)^{T}$; the initial value of the identification is $P(0)=10^{6} \times I_{5 \times 5}$ ( $I$ is the unit matrix), and the forgetting factor $\lambda=0.998$.

Step3: Calculate $\varphi(k)$ on the basis of the current observation data $V(k-1)-V_{o c}(k-1), V(k-2)-V_{o c}(k-2), I(k), I(k-1), I(k-2), V_{o c}(k)-V(k)$.

Step4: Calculate $\hat{\theta}(k) 、 K(k)$ and $P(k)$ on the basis of Eq.(2-7).

Step5: Let $k=k+1$.If $k \leq n$, go to Step2, otherwise, end the algorithm, and $\hat{\theta}=\hat{\theta}(n)$.

\subsection{Analysis of model parameter identification}

In Beijing, each taxi BEV produced by the Motor Corporation is composed of 100 lithium iron phosphate battery packs in series, and each pack consists of two parallel cells. The data used in this study are collected from eight electric taxis on August 23, 2015. The data include time, vehicle ID, total voltage, total current, SOC, temperature. The data sampling period is $5 \mathrm{~s}$, and the sample size is 970 . In previous experiments, we found that compared with the electric vehicle discharging process, the charging current of the battery pack is relatively stable, and the charging voltage does not fluctuate frequently. Thus, the model parameters identification is confirmed on the basis of the charging data of BEVs.

According to the identification approach described in Section 2.2, the parameters in second-order RC equivalent circuit battery model are identified, and the testing results are shown in Fig. 2. 

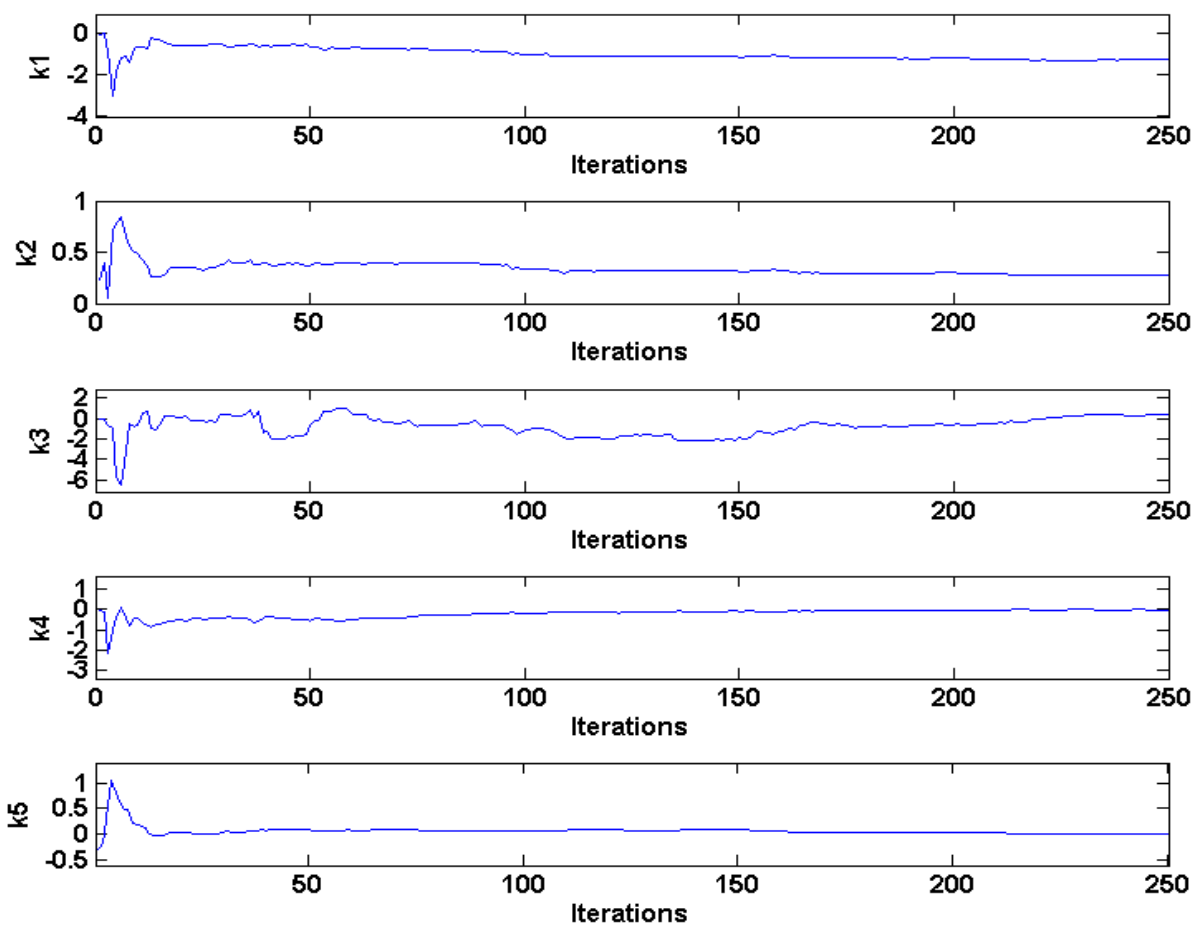

Fig. 2. Identification curve

All the curves exhibit a small fluctuation during parameter identification. After 200 iterations, all the curves tend to converge. After convergence, all parameters $k$ can be acquired.

The results of parameter $k$ identification are provided in Table 1 , and the corresponding solution parameters $R_{0}, R_{1}, R_{2}, C_{1}, C_{2}, \tau_{1}, \tau_{2}$ are presented in Table 2.

Table 1. Results of parameter k identification

\begin{tabular}{cccccc}
\hline \multirow{2}{*}{ Vehicle ID } & \multicolumn{5}{c}{ Parameters } \\
\cline { 2 - 6 } & $k_{1}$ & $k_{2}$ & $k_{3}$ & $k_{4}$ & $k_{5}$ \\
\hline 1 & -1.1278 & 0.3071 & 0.0701 & 0.0676 & 0.0151 \\
2 & -1.217 & 0.3411 & 0.07195 & -0.075 & 0.01685 \\
3 & -1.2488 & 0.3698 & 0.07581 & -0.07631 & 0.01846 \\
4 & -1.2274 & 0.3607 & 0.7339 & -0.0778 & 0.0178 \\
5 & -1.1689 & 0.334 & 0.075 & -0.07298 & 0.0167 \\
6 & -1.1483 & 0.3012 & 0.0804 & -0.076 & 0.0146 \\
7 & -1.1577 & 0.3144 & 0.0813 & -0.0785 & 0.0158 \\
8 & -1.1967 & 0.3257 & 0.0711 & -0.0731 & 0.0162 \\
\hline
\end{tabular}

Table 2. Values of model parameters

\begin{tabular}{clllllll}
\hline Vehicle & \multicolumn{7}{c}{ Parameters } \\
\cline { 2 - 7 } ID & $R_{1}(\mathrm{~m} \Omega)$ & $R_{2}(\mathrm{~m} \Omega)$ & $C_{1}(\mathrm{~F})$ & $C_{2}(\mathrm{~F})$ & $\tau_{1}(\mathrm{~s})$ & $\tau_{2}(\mathrm{~s})$ & $R_{0}(\mathrm{~m} \Omega)$
\end{tabular}




\begin{tabular}{llllllll}
\hline 1 & 25.3 & 23.5 & 167.98 & 428.51 & 4.25 & 10.07 & 48.8 \\
2 & 23.2 & 37.4 & 202.16 & 389.04 & 7.01 & 14.55 & 49.4 \\
3 & 32.2 & 34.6 & 178.15 & 261.82 & 5.11 & 14.02 & 49.9 \\
4 & 31.5 & 21.9 & 114.71 & 295.27 & 4.756 & 14.22 & 49.3 \\
5 & 24 & 39.4 & 206.17 & 259.52 & 4.948 & 10.22 & 50 \\
6 & 36.9 & 38.9 & 92.51 & 370.96 & 3.41 & 14.44 & 48.8 \\
7 & 31.9 & 26.5 & 91.81 & 491.54 & 3.851 & 13.025 & 50.3 \\
8 & 29.1 & 27.6 & 155.3 & 302.9 & 4.859 & 12.45 & 50.1 \\
\hline
\end{tabular}

Referring to [43-46],the internal resistance of the battery pack (including the ohmic resistance and polarization resistance) of a BEV generally ranges from 100 $\mathrm{m} \Omega$ to $150 \mathrm{~m} \Omega$, and the ohmic resistance ranges from $45 \mathrm{~m} \Omega$ to $55 \mathrm{~m} \Omega$. According to the manufacturers of the eight BEVs, the initial statistic of battery pack internal resistance also ranges from $45 \mathrm{~m} \Omega$ to $55 \mathrm{~m} \Omega$. On the basis of the parameter identification results, we can calculate the battery internal resistance $R_{0}+R_{1}+R_{2}$ and the ohmic resistance $R_{0}$ (Table 3 ). The values are basically located within a reasonable range.

Table 3. Values of resistance

\begin{tabular}{ccccccccc}
\hline \multirow{2}{*}{ Resistance } & \multicolumn{7}{c}{ Vehicle ID } \\
\cline { 2 - 9 } & 1 & 2 & 3 & 4 & 5 & 6 & 7 & 8 \\
\hline $\mathrm{R} 0+\mathrm{R} 1+\mathrm{R} 2(\mathrm{~m} \Omega)$ & 98 & 110 & 117 & 102 & 113 & 124 & 109 & 100 \\
$\mathrm{R} 0(\mathrm{~m} \Omega)$ & 49 & 49 & 50 & 49 & 50 & 49 & 50 & 50 \\
\hline
\end{tabular}

\section{Battery pack SOH estimation theory}

\subsection{Battery pack SOH of BEV}

Internal resistance dynamically changes during battery processing. The relationship between $\mathrm{SOH}$ and ohmic resistance is strong. Therefore, battery internal resistance is adopted to reflect the $\mathrm{SOH}$ of the battery system. $\mathrm{SOH}$ is defined as (3-1)

$$
S O H=\frac{R_{E O L}-R_{\text {now }}}{R_{\text {EOL }}-R_{\text {new }},}
$$

where $R_{E O L}$ is the resistance when the battery life ends, $R_{n e w}$ is the current Ohmic resistance, and $R_{\text {new }}$ is the original Ohmic resistance[47]. The SOH ranges from $0 \%$ to $100 \%, 100 \%$ implies the battery is new, whereas $0 \%$ indicates that the battery should be replaced.

In an electrochemical reaction, battery $\mathrm{SOH}$ declines following the increasing of the internal resistance. According to[48], when the internal resistance increases by $25 \%$, battery failure may occur; when the internal resistance increases by $50 \%$, the battery has a serious fault; when the internal resistance increases by $100 \%$ or more, 
the battery fails. Thus, battery internal resistance $R$ can be used as an evaluation index of the Li-ion battery SOH. Consequently, this research assumes $R_{E O L}=1.6 \times R_{\text {new }}$.

\subsection{State equation of SOH estimation}

To track and predict battery $\mathrm{SOH}$, we develop a state-space estimation model. Given that a battery charges slowly, the battery internal resistance can be regarded as a constant in most charging processes. When establishing the change in the state equation of internal resistance, we can adopt a small noise disturbance to represent the resistance change in the charging process. To estimate the resistance, we use voltage $V_{k}$ as the observation variable. The state equation and observation equation of the battery internal resistance (see Fig. 1) are as follows:

$$
\begin{gathered}
R_{0, k+1}=R_{0, k}+\gamma_{k} \\
V_{k}=V_{o c}-i_{k} R_{0, k}-V_{k}^{R_{1} C_{1}}-V_{k}^{R_{2} C_{2}}+\eta_{k}
\end{gathered}
$$

Eqs. (3-2) and (3-3) describe the dynamic change in the battery internal resistance; here, a small disturbance $\gamma_{k}$ describes the slow change in the internal resistance in a charging process, $\eta_{k}$ is the measurement noise, $i_{k}$ is the input current of the system.

The equation of the $R_{1} C_{1}$ parallel loop in the Fig. 1is defined as Eq. (3-4), where $V_{k}^{R_{1} C_{1}}$ is the voltage of the $R_{1} C_{1}$ parallel loop and $i_{s 1}$ is the current of the $R_{1} C_{1}$ parallel loop.

$$
V_{k}^{R_{1} C_{1}}=V_{k}^{R_{1} C_{1}} e^{-\frac{t}{\tau_{1}}}+R_{1} i_{s 1}\left(1-e^{-\frac{t}{\tau_{1}}}\right)
$$

The equation of the $R_{2} C_{2}$ parallel loop is defined as Eq. (3-5), where $V_{k}^{R_{2} C_{2}}$ and $i_{s 2}$ are the voltage and current of the $R_{2} C_{2}$ parallel loop, respectively.

$$
V_{k}^{R_{2} C_{2}}=V_{k}^{R_{2} C_{2}} e^{-\frac{t}{\tau_{2}}}+R_{2} i_{s 2}\left(1-e^{-\frac{t}{\tau_{2}}}\right)
$$

To discretize $V^{R_{1} C_{1}}$ and $V^{R_{2} C_{2}}$,

$$
\begin{gathered}
V_{k+1}^{R_{1} C_{1}}=V_{k}^{R_{1} C_{1}} e^{-\frac{\Delta t}{\tau_{1}}}+R_{1} i_{k}\left(1-e^{-\frac{\Delta t}{\tau_{1}}}\right)+\omega_{k}, \\
V_{k+1}^{R_{2} C_{2}}=V_{k}^{R_{2} C_{2}} e^{-\frac{\Delta t}{\tau_{2}}}+R_{2} i_{k}\left(1-e^{-\frac{\Delta t}{\tau_{2}}}\right)+\omega_{k},
\end{gathered}
$$

where $\Delta t$ is the sampling period and $\omega_{k}$ is the measurement noise of $V_{k}^{R_{1} C_{1}}$ and $V_{k}^{R_{2} C_{2}}$.

\section{$3.3 \mathrm{SOH}$ estimation based on genetic resampling particle filter algorithm}

\subsubsection{Particle filter algorithm}

The $\mathrm{PF}$ is a random track estimation algorithm, which is also effective algorithm in solving the issues of nonlinear correlation. To solve the non-Gaussian noise 
produced by simplifying the battery pack model, we use the PF in estimating resistance in this study. The PF, through a group random samples or "particles" which constantly update according to the Bayesian rules, estimates posterior probability density of an unknown state[49]. In this study the resistance is represented by a particle set. These samples (or "particles") are obtained by sampling the posterior density on the basis of sequential importance. These particles comprise a set of corresponding weights.

The steps of standard PF algorithm are as follows [31]:

First, parameters are defined as follows:

$x_{k}$ is the target state sequence ;

$\left\{x_{0: k}^{i}\right\}_{i=1}^{N_{s}}$ is the particles set, which represents the resistance $\mathrm{R}_{0}$ in this study.

$\mathrm{Z}_{k}$ is the observation sequence that represents the voltage $V_{k}$ in this study.

$p\left(x_{0}\right)$ is a priori conditional probability of the dynamic system state.

$p\left(x_{0: k} \mid z_{1: k}\right)$ is the posterior probability distribution.

$\left\{\omega_{k}^{i}, i=0, \cdots, N_{s}\right\}$ is the weight that is normalized as $\sum_{i} \omega_{k}^{i}=1$.

$\left\{x_{0: k}^{i}, \omega_{k}^{i}\right\}_{i=1}^{N_{s}}$ describes the posterior probability distribution of the target state $x_{k}$ at $k$ moment.

$x_{0: k}=\left\{x_{j}, j=0, \cdots, k\right\}$ is the set of states from 0 to $k$ moment.

$q\left(x_{0: k} \mid z_{1: k}\right)$ is the importance density function.

The discrete-time dynamic stochastic model of the system is defined as

$$
\begin{gathered}
x_{k}=f_{k}\left(x_{k-1}, \varpi_{k-1}\right), \\
z_{k}=h_{k}\left(x_{k}, v_{k-1}\right) .
\end{gathered}
$$

Then the posterior probability distribution of the target state can be discretely weighted as

$$
p\left(x_{0: k} \mid z_{1: k}\right) \approx \sum_{i=1}^{N_{s}} \omega_{k}^{i} \delta\left(x_{0: k}-x_{0: k}^{i}\right),
$$

in which the weights are selected according to the importance sampling approach.

The importance density function is defined as:

$$
q\left(x_{0: k} \mid z_{1: k}\right)=q\left(x_{k} \mid x_{0: k-1}, z_{1: k}\right) q\left(x_{0: k-1} \mid z_{1: k-1}\right) .
$$

If the particle set can be obtained by the importance density function, we can define the weight as:

$$
\omega_{k}^{i} \infty \frac{p\left(x_{0: k} \mid z_{1: k}\right)}{q\left(x_{0: k} \mid z_{1: k}\right)}
$$

Thus, particles $\left\{x_{k}^{i}\right\}_{i=1}^{N_{s}}$ are acquired from $q\left(x_{k} \mid x_{0: k-1}, z_{1: k}\right)$, particle set $\left\{x_{0: k-1}^{i}\right\}_{i=1}^{N_{s}}$ is acquired from $q\left(x_{0: k-1} \mid z_{1: k-1}\right)$, and the new particle set $\left\{x_{0: k}^{i}\right\}_{i=1}^{N_{s}}$ can be acquired. 
The posterior probability density function can be expressed as

$$
p\left(x_{0: k} \mid z_{1: k}\right) \infty p\left(z_{k} \mid x_{k}\right) p\left(x_{k} \mid x_{k-1}\right) p\left(x_{0: k-1} \mid z_{1: k-1}\right) .
$$

By substituting Eqs. (3-11) and (3-12) into Eq. (3-13), the updated formula for the importance weights can be expressed as

$$
\omega_{k}^{i} \infty \frac{p\left(z_{k} \mid x_{k}^{i}\right) p\left(x_{k}^{i} \mid x_{k-1}^{i}\right) p\left(x_{0: k-1}^{i} \mid z_{1: k-1}\right)}{q\left(x_{k}^{i} \mid x_{0: k-1}^{i}, z_{1: k}\right) q\left(x_{0: k-1}^{i} \mid z_{1: k-1}\right)}=\omega_{k-1}^{i} \frac{p\left(z_{k} \mid x_{k}^{i}\right) p\left(x_{k}^{i} \mid x_{k-1}^{i}\right)}{q\left(x_{k}^{i} \mid x_{0: k-1}^{i}, z_{1: k}\right)} .
$$

The standard PF algorithm adopts the prior probability density as the importance density function.

$$
q\left(x_{k}^{i} \mid x_{k-1}^{i}, z_{k}\right)=p\left(x_{k}^{i} \mid x_{k-1}^{i}\right) .
$$

By substituting the Eq. (3-14) into the Eq. (3-15), the importance weight can be simplified as

$$
\omega_{k}^{i} \infty \omega_{k-1}^{i} p\left(z_{k} \mid x_{k}^{i}\right)
$$

Weight $\omega_{k}^{i}$ is normalized as

$$
\omega_{k}^{i}=\omega_{k}^{i} / \sum_{i=1}^{N_{s}} \omega_{k}^{i}
$$

The posterior probability density function $p\left(x_{k} \mid z_{1: k}\right)$ is defined as

$$
p\left(x_{k} \mid z_{1: k}\right) \approx \sum_{i=1}^{N_{s}} \omega_{k}^{i} \delta\left(x_{k}-x_{k}^{i}\right) \text {. }
$$

According to the law of large numbers, Eq. (3-18) can approximate the real posterior probability $p\left(x_{k} \mid z_{1: k}\right)$ when $N_{s} \rightarrow \infty$.

\subsubsection{Genetic resampling particle filter algorithm}

The degradation problem in the PF algorithm seriously influences the estimation accuracy. Only a small number of particles have large weights, whereas most particles have negligible weights. Thus, the abundant recursive calculation is wasted on the updates of particles, which almost do not work. To increase the number of meaningful particles and guarantee the diversity of the particle set, we propose resampling for the degradation problem [50]. The genetic algorithm is an intelligent optimization that can produce a new individual and ensure the superiority of the population. Thus, the genetic algorithm in the PF algorithm is adopted to improve the diversity of the particle set and thereby increase the estimation accuracy of battery pack internal resistance.

The GPF emphatically incorporates evolutionary thinking (i.e., selection, crossover, and mutation) into the PF algorithm rather than traditional resampling methods to optimize the algorithm[Error! Bookmark not defined.]. The genetic algorithm has a special search optimization ability, which can reduce the number of particles close to the maximum of the posteriori probability distribution. Therefore, the genetic algorithm can reduce the computation to improve the efficiency of particle 
performance. Moreover, the genetic algorithm can effectively increase the diversity of particles and solve the particle degradation problem to improve the accuracy of state estimation.

The specific steps of the selection, crossover and mutation operations in the GPF are as follows:

(1) Selection operation. Define the weighted particle set at $k$ moment as $\left\{x_{k}^{i}, \omega_{k}^{i}\right\}_{i=1}^{N_{s}}$. Define $\omega_{k}^{i}$ as the population adaptability of the particles in the particle set. Then, calculate the fitness variance, which is the variance of the importance weights of the particles. When the variance of the importance weights is minimal, the state estimation is closest to the true state. In this case, adapt the variance to determine whether to implement selection operation. If the variance meets the requirements, perform particle selection operation, keep all the particles, and directly proceed to the estimation phase. Otherwise, skip the selection operation and directly proceed to crossover and mutation operations.

(2) Crossover operation. Randomly select two particles in the particle set, and perform the crossover operation according to the Eqs. (3-19) and (3-20).

$$
\begin{aligned}
& \tilde{x}_{k}^{m}=\alpha x_{k}^{m}+(1-\alpha) x_{k}^{n}+\eta, \\
& \tilde{x}_{k}^{n}=\alpha x_{k}^{n}+(1-\alpha) x_{k}^{m}+\eta,
\end{aligned}
$$

where , $\quad \eta \sim N(0, \Sigma), \quad \alpha \sim U(0,1)$, and $N(0, \Sigma)$ obey a normal distribution, whereas $U(0,1)$ obeys a uniform distribution. The cross rule implies that if $p\left(z_{k} \mid \tilde{x}_{k}^{m}\right)>\max \left\{p\left(z_{k} \mid x_{k}^{m}\right), p\left(z_{k} \mid x_{k}^{n}\right)\right\}$ then the particle must be adopted; otherwise, the particle whose probability is $p\left(z_{k} \mid \tilde{x}_{k}^{m}\right) / \max \left\{p\left(z_{k} \mid x_{k}^{m}\right), p\left(z_{k} \mid x_{k}^{n}\right)\right\}$ is adopted. The methods of accepting and abandoning particle $\tilde{x}_{k}^{n}$ are the same as those for $\tilde{x}_{k}^{m}$.

(3) Mutation operation. Randomly select a particle from the particle set, and perform the mutation process according to Eq. (3-21)

$$
\tilde{x}_{k}^{j}=x_{k}^{j}+\eta, \quad \eta \sim N(0, \Sigma) .
$$

The mutation rule is that if $p\left(z_{k} \mid \tilde{x}_{k}^{j}\right)>p\left(z_{k} \mid x_{k}^{j}\right)$, then the particle must be adopted; otherwise, the particle whose probability is $p\left(z_{k} \mid \tilde{x}_{k}^{j}\right) / p\left(z_{k} \mid x_{k}^{j}\right)$ is adopted.

According to GPF theory, the specific steps for battery internal resistance estimation based on the steps of the GPF are as follows:

Step 1: Initialization. Set the value of $V_{0}^{R_{1} C_{1}}$ and $V_{0}^{R_{2} C_{2}}$ to 0 , calculate $V^{R_{1} C_{1}}$ and $V^{R_{2} C_{2}}$ on the basis of Eqs. (3-8) and (3-9), respectively. The initial iteration variable $k$ is equal to 0 . The iteration is $N$; according to a previous work, $N$ is equal to 1,000 , and the particle number $M$ is equal to 300 .

Step 2: Obtain the particle set that contains $M$ particles by sampling based on the random normal probability density function. Let the particle initial weights be equal to $1 / M$. 
Step 3: Use the battery internal resistance state equation (3-10) to calculate the particles value, and use the observation equation (3-11) to calculate $V_{k}^{i}$ corresponding to $x_{k}^{i}$.

Step 4: Update particle weights. Calculate each particle weight $\omega_{k}^{i}$ on the basis of $\omega_{k}^{i}=\frac{1}{\sqrt{2 \pi} s} e^{-\frac{\left(V_{k}-V_{k}^{i}\right)^{2}}{2 s^{2}}}$, where $s$ is the standard deviation of the noise. Then, normalize $\omega_{k}^{i}$ on the basis of $\omega_{k}^{i}=\omega_{k}^{i} / \sum_{i}^{M} \omega_{k}^{i}$.

Step 5 : According to $N_{\text {eff }}=1 / \sum_{i}^{M}\left(\omega_{k}^{i}\right)^{2}<N_{\text {threshold }}$, determine whether the particle set performs the genetic resampling operation. If genetic resampling is necessary, then proceed to Step6. If not, keep all particles obtained from the Step3, and perform Step9.

Step 6 : Take importance weights $\omega_{k}^{i}\{i=1,2, \cdots M\}$ as individual fitness function values $f_{k}^{i}=\omega_{k}^{i}\{i=1,2, \cdots M\}$, and then set the crossover probability $p_{c}$ and mutation probability $p_{m}$ for the algorithm.

Step 7: According to the predetermined crossover probability and mutation probability, perform the crossover and mutation operations on the basis of Eqs.(3-19), (3-20) and (3 - 21).

Step 8: Using gambling to optimize particles after genetic manipulation, obtain particles $\left\{x_{k}^{i}, \omega_{k}^{i}\right\}_{i=1}^{M}$.

Step 9: Determine whether to reach the amount of population evolution. If it is reached then proceed to Step 11; otherwise, return to Step 6.

Step 10 : Evaluate the state, and calculate its value $\hat{x}_{k}=\sum_{i=1}^{M} \omega_{k}^{i} x_{k}^{i}$.

Step 11 : Determine whether to terminate the algorithm. If $k=N$, then exit the algorithm; otherwise, let $k=k+1$, and return to Step 3 .

The flowchart of GPF is shown in Fig.3. 


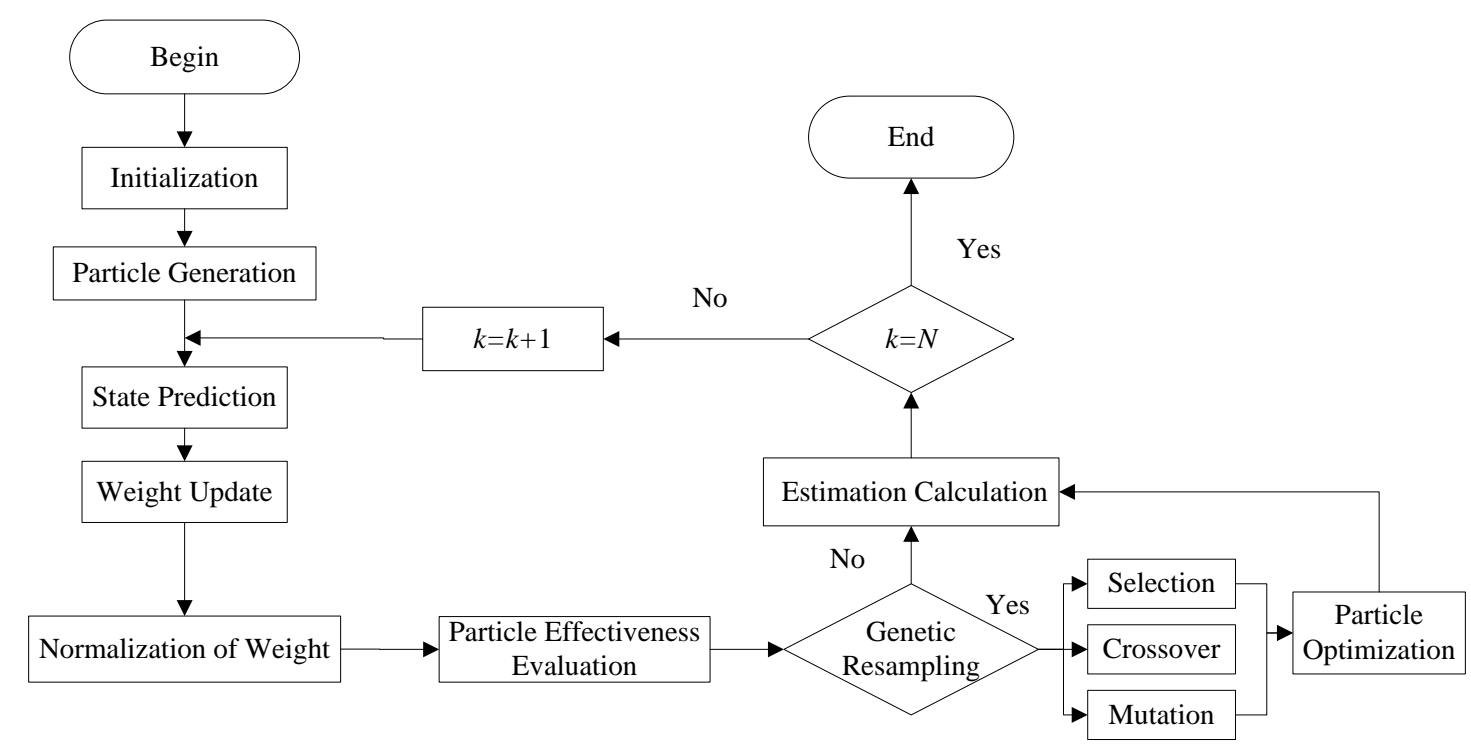

Fig3. Flowchart of GPF

\section{Experiment and analysis}

\subsection{Estimation results and analysis based on genetic resampling particle filter}

We carry out the experiment according to the specific steps of the GPF in Section 3.3. The experimental data are the same as those used in Section 2. Set $R_{E O L}=1.6 * R_{\text {new }}$. According to Table $2, R_{1}$ is equal to the mean of $\mathrm{R}_{1}$ in the battery packs of the eight vehicles. We obtain $R_{2}, \tau_{1}$, and $\tau_{2}$ similarly; thus, $R_{1}=31.4 \mathrm{~m} \Omega, R_{2}=30.2 \mathrm{~m} \Omega, \tau_{1}=3.978 \mathrm{~s}$, and $\tau_{2}=11.757 \mathrm{~s}$. Let the initial value of $R_{0}$ be equal to $50 \mathrm{~m} \Omega$ according to Table 3 , and let $V_{1}^{R_{1} C_{1}}=-0.2443 V$ and $V_{1}^{R_{2} C_{2}}=-0.1793 V$. The results are demonstrated in Figs. 4 and 5 . 


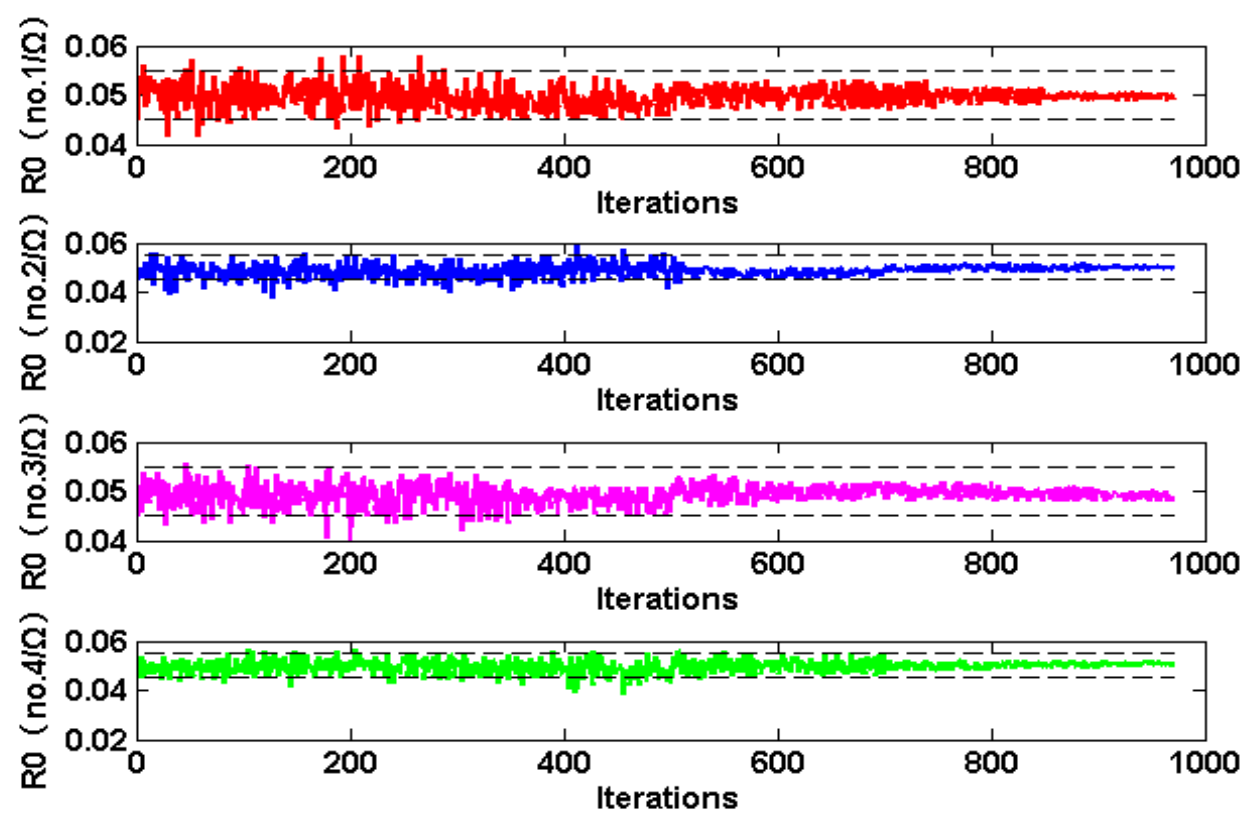

Fig. 4. Internal resistance curves of electric taxis 1, 2, 3, and4
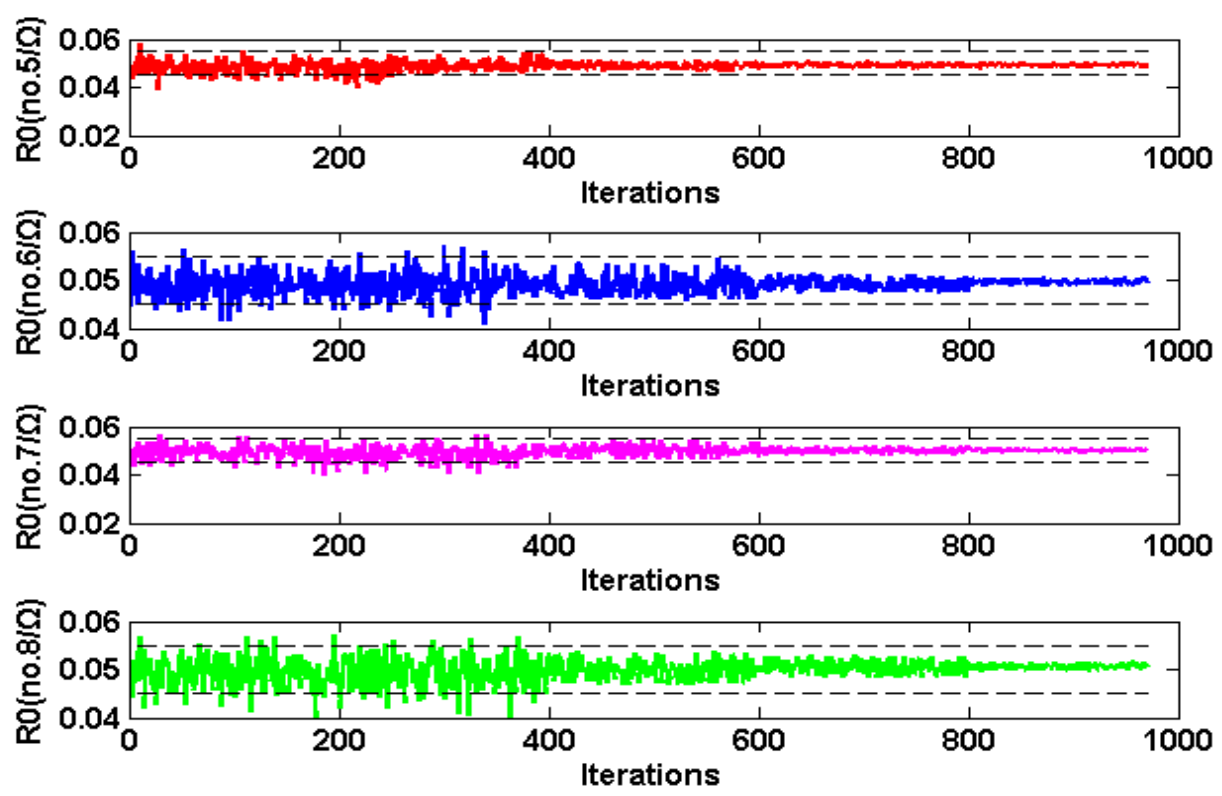

Fig. 5. Internal resistance curves of electric taxi5, 6, 7, and 8

The two black dotted lines represent the values of 45 and $55 \mathrm{~m} \Omega$. From the figures, the curves exhibit good stability, and no large fluctuation is evident during estimation. As the iterations increase, the width of the curve fluctuation decreases. In Table 4, the experimental results during the iterative process are presented in detail.

Table 4. Experimental result statistics of estimation for internal resistance

\begin{tabular}{ccccccc}
\hline Vehicle & \multicolumn{7}{c}{ Records of $\mathrm{R}_{0}(\mathrm{~m} \Omega)$} \\
\cline { 2 - 7 } ID & $20-40$ & $40-45$ & $45-55$ & $55-60$ & $60-70$ & $70-100$ \\
\hline 1 & 0 & 11 & 949 & 10 & 0 & 0
\end{tabular}




\begin{tabular}{ccccccc}
2 & 4 & 61 & 898 & 7 & 0 & 0 \\
3 & 0 & 26 & 942 & 2 & 0 & 0 \\
4 & 2 & 35 & 914 & 19 & 0 & 0 \\
5 & 1 & 38 & 929 & 2 & 0 & 0 \\
6 & 0 & 29 & 934 & 7 & 0 & 0 \\
7 & 1 & 40 & 924 & 5 & 0 & 0 \\
8 & 0 & 30 & 929 & 11 & 0 & 0 \\
\hline
\end{tabular}

Table 4 reveals that most of the results are located within the reasonable interval of $[45,55 \mathrm{~m} \Omega]$. Hence, the GPF algorithm achieves a high performance for dynamic battery internal resistance estimation.

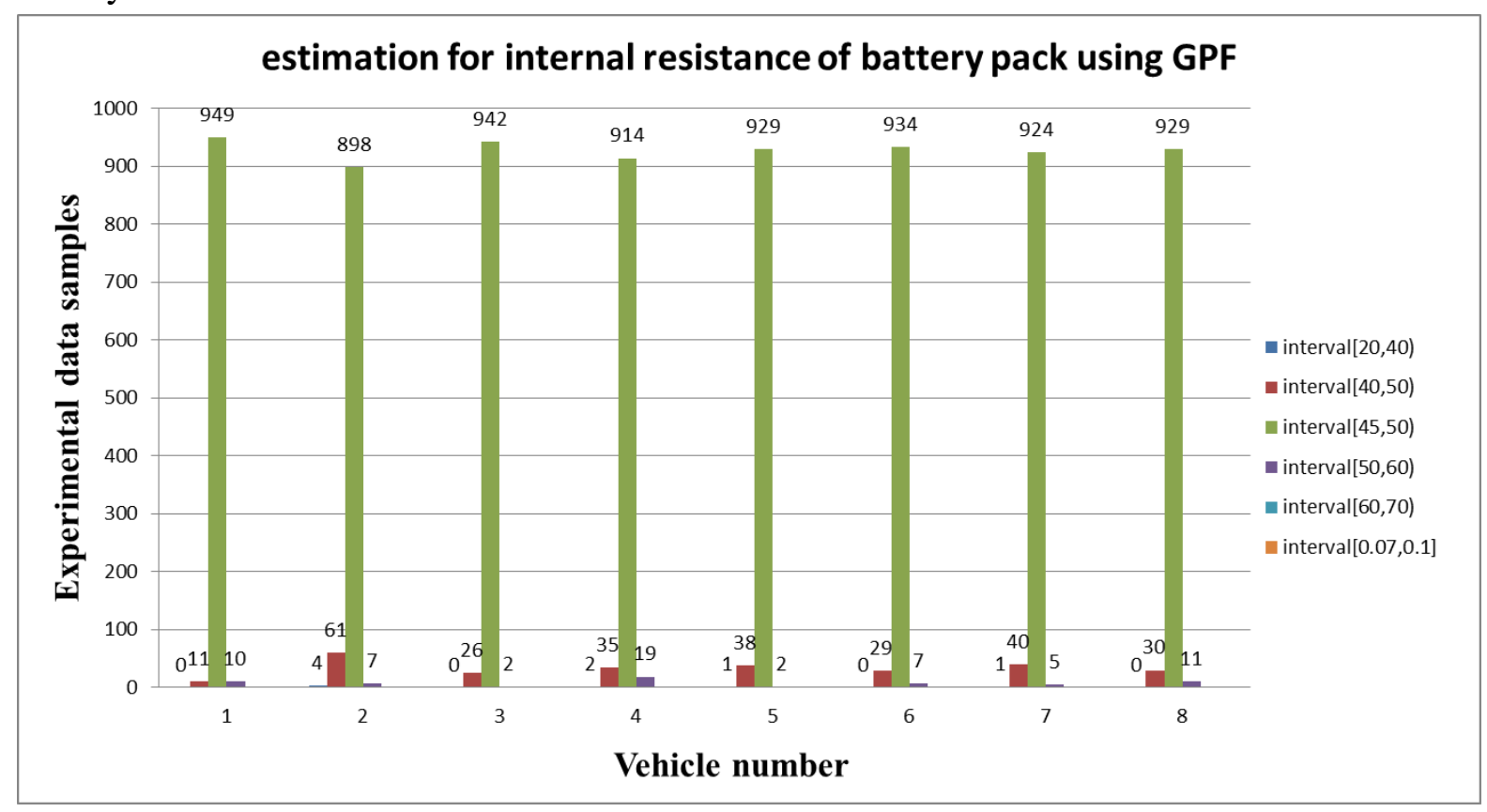

Fig. 6. Experimental result analysis of estimation for internal resistance

The histograms (Fig. 6) intuitively describe the experimental results based on the GPF algorithm, and are distribute in different intervals. Unlike that of the green rectangles, the height and interval span of the rectangles in other color are smaller, which indicates that the experimental results are mainly in the $[45,55 \mathrm{~m} \Omega]$ interval. In addition, the data fluctuation is small and the change trend is relatively stable in the experiment. Therefore, the experimental results confirm that the GPF can be effectively used to estimate battery internal resistance.

\subsection{Comparison of algorithm accuracy and practicability}

To prove that the GPF algorithm can more effectively estimate SOH than the PF algorithm, we compare the results of the PF and GPF algorithms. The contrastive histograms in Fig. 7 demonstrate that the experimental results of the GPF and PF are mostly in the $[45,55 \mathrm{~m} \Omega$ ] interval. However, the battery internal resistance estimation of the GPF algorithm in this interval is significantly greater than that of the PF algorithm. The contrastive results illustrate the relative superiority of the GPF. 


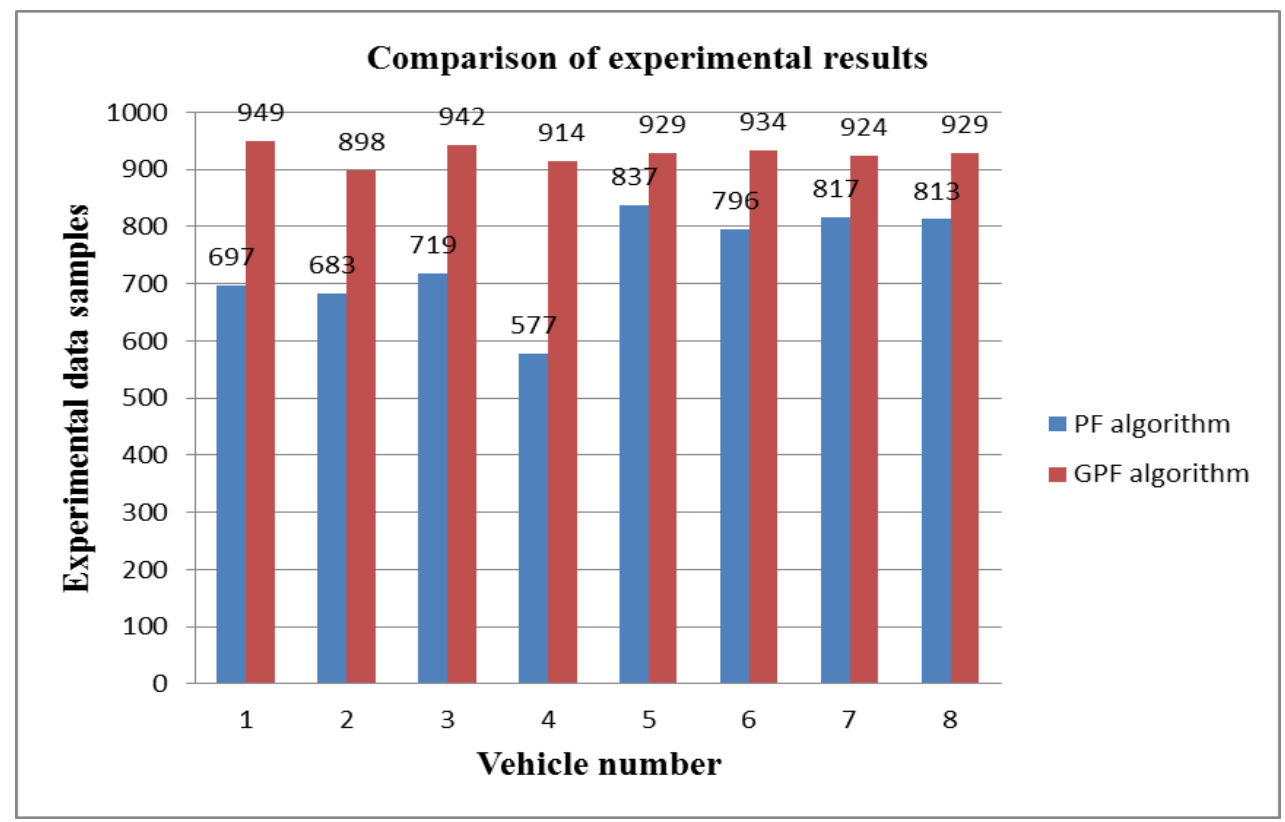

Fig. 7. Comparison of estimation results for internal resistance by GPF and PF

Table 5 presents the comparative analysis of the PF and GPF experimental results. Quantitatively, the percentage of resistance solved by the GPF algorithm is significantly higher than that solved by the PF algorithm in the $[45,55 \mathrm{~m} \Omega$ ] interval.

Tab. 5. Statistical experimental results for internal resistance estimation by GPF and PF

\begin{tabular}{ccccccc}
\hline \multirow{2}{*}{ Vehicle ID } & \multicolumn{6}{c}{ Distribution percentage of $\mathrm{R}_{0}(\mathrm{~m} \Omega)$} \\
\cline { 2 - 7 } $\mathbf{1}$ & $20-40$ & $40-45$ & $45-55$ & $55-60$ & $60-70$ & $70-100$ \\
\hline \multirow{2}{*}{$\mathbf{2}$} & $5.46 \%$ & $4.33 \%$ & $71.86 \%$ & $7.11 \%$ & $10.11 \%$ & $1.13 \%$ \\
& 0 & $1.13 \%$ & $97.84 \%$ & $1.03 \%$ & 0 & 0 \\
\multirow{3}{*}{$\mathbf{3}$} & $0.31 \%$ & $1.03 \%$ & $70.41 \%$ & $16.49 \%$ & $11.34 \%$ & $0.41 \%$ \\
& $0.41 \%$ & $6.29 \%$ & $92.58 \%$ & $0.72 \%$ & 0 & 0 \\
\multirow{4}{*}{} & $0.1 \%$ & $0.41 \%$ & $74.12 \%$ & $16.8 \%$ & $8.56 \%$ & 0 \\
& $0.1 \%$ & $2.68 \%$ & $97.11 \%$ & $0.21 \%$ & 0 & 0 \\
\multirow{5}{*}{} & $0.21 \%$ & $3.61 \%$ & $94.22 \%$ & $1.96 \%$ & 0 & 0 \\
& $1.96 \%$ & $5.46 \%$ & $86.29 \%$ & $5.15 \%$ & $1.13 \%$ & 0 \\
$\mathbf{6}$ & $0.1 \%$ & $3.92 \%$ & $95.77 \%$ & $0.21 \%$ & 0 & 0 \\
& $2.16 \%$ & $6.81 \%$ & $82.06 \%$ & $7.01 \%$ & $1.96 \%$ & 0 \\
\multirow{7}{*}{} & 0 & $2.99 \%$ & $96.29 \%$ & $0.72 \%$ & 0 & 0 \\
& $1.44 \%$ & $6.6 \%$ & $84.22 \%$ & $5.77 \%$ & $1.86 \%$ & $0.1 \%$ \\
$\mathbf{8}$ & $0.1 \%$ & $4.12 \%$ & $95.26 \%$ & $0.52 \%$ & 0 & 0 \\
& $2.27 \%$ & $5.67 \%$ & $83.81 \%$ & $6.6 \%$ & $1.65 \%$ & 0 \\
& 0 & $3.09 \%$ & $95.78 \%$ & $0.52 \%$ & 0 & 0 \\
\hline
\end{tabular}

Note: The black and red parts represent the PF and GPF algorithms, respectively.

As indicated in the variance of the experimental results in Table 6, the values solved by the GPF algorithm are evidently smaller than those solved by the PF algorithm. Hence, the experimental results of GPF algorithm show minimal fluctuation. In sum, the GPF algorithm is more accurate and applicable in battery pack 
resistance estimation than the PF algorithm.

Table 6. Comparison of the variance in the experimental results of PF and GPF

\begin{tabular}{ccccccccc}
\hline \multirow{2}{*}{ Algorithm } & \multicolumn{8}{c}{ Variance } \\
\cline { 2 - 9 } & 1 & 2 & 3 & 4 & 5 & 6 & 7 & 8 \\
\hline PF & 6.2 & 2.37 & 2.2 & 2.55 & 1.37 & 1.76 & 1.64 & 1.55 \\
GPF & 0.49 & 0.63 & 0.4 & 0.64 & 0.35 & 0.42 & 0.49 & 0.53 \\
\hline
\end{tabular}

\subsection{SOH estimation based on resistance}

In reference to previous works, the battery pack initiative resistance $R_{\text {neW }}=45 \mathrm{n} \Omega$. According to Section 3.1, $R_{\text {new }}=72 n \Omega$. The SOH estimation results of the eight taxi BEVs based on Eq. (3-1) are provided in Table 7.

Table 7. Results of battery pack SOH estimation

\begin{tabular}{ccccccccc}
\hline & \multicolumn{8}{c}{ Values } \\
\cline { 2 - 9 } Parameters & 1 & 2 & 3 & 4 & 5 & 6 & 7 & 8 \\
\hline \multirow{2}{*}{$\mathrm{R}_{0}$} & 47.57 & 48.74 & 48.22 & 48.57 & 47.82 & 48.04 & 47.59 & 47.83 \\
$\mathrm{SOH}$ & $90.48 \%$ & $86.15 \%$ & $88.07 \%$ & $86.78 \%$ & $89.56 \%$ & $88.74 \%$ & $90.48 \%$ & $86.15 \%$ \\
\hline
\end{tabular}

The battery pack SOH of the eight taxi BEVs is above $80 \%$, which indicates that the electric taxi battery packs are healthy. Most results are below 90\%, which implies that the taxi BEVs have been used for a long time; such condition is close to actual working conditions.

\section{Conclusions}

In this paper, ohmic resistance is employed as the evaluation index of battery pack $\mathrm{SOH}$ to express the power output. To measure the internal resistance, we establish a second-order RC battery equivalent circuit model, whose unknown parameters are identified with the recursive least squares method with forgetting factor. A battery system is strongly nonlinear and non-Gaussian. Non-Gaussian errors occur when the model is simplified to reduce the calculation complexity. Given that the GPF is effective in solving nonlinear and non-Gaussian problems; it is adopted to estimate the $\mathrm{SOH}$ in this study. On the basis of real charging data, we carry out estimation experiments on battery pack internal resistance. Finally, the comparison of the experimental results shows that the GPF algorithm is more accurate and applicable in estimating battery pack internal resistance than the PF algorithm. In addition, the experiments are conducted according to the real data collected from electric taxis in Beijing. This study can provide a new perspective for BEV SOH estimation. Moreover, as indicated by the research results, BEV drivers can clearly understand 
battery $\mathrm{SOH}$ to maintain or replace battery packs in time.

Charging data are used in all the experiments. Thus, future studies should use discharging data to improve research. The existing knowledge on open-circuit voltage and resistance is obtained from previous works. Considering that the practical state of battery packs changes dynamically, future research should establish an adaptive system according to the correct model parameters of real-time battery data.

\section{Acknowledge}

This paper is sponsored the National Science Foundation of China for the Central Universities (71471014).

\section{References}

[1] Sepasi S, Roose L R, Matsuura M M. Extended Kalman Filter with a Fuzzy Method for Accurate Battery Pack State of Charge Estimation[J]. Energies, 2015, 8(6):5217-5233.

[2] Marongiu A, Marco R, Sauer DU. Influence of the vehicle-to-grid strategy on the aging behavior of lithium battery electric vehicles. Appl Energy 2015;137:899-912.

[3] Wang Y, Zhang C, Chen Z, Xie J, Zhang X. A novel active equalization method for lithium-ion batteries in electric vehicles. Appl Energy 2015;145:36-42.

[4] Uddin K, Picarelli A, Lyness C, et al. An Acausal Li-Ion Battery Pack Model for Automotive Applications[J]. Energies, 2014, 7(9):5675-5700.

[5] Feng X, Li J, Ouyang M, et al. Using probability density function to evaluate the state of health oflithium-ion batteries[J]. Journal of Power Sources, 2013, 232(18):209-218.

[6] Chen Z, Mi C C, Fu Y, et al. Online battery state of health estimation based on Genetic Algorithm for electric and hybrid vehicle applications $\hat{\imath}[\mathrm{J}]$. Journal of Power Sources, 2013, 240(31):184-192.

[7] Wenjing, Xu. Study on State of Health Estimation Algorithm for Lithium Power Battery Used on Pure Electric Vehicle[D]. Jilin:Jilin University, 2012.

[8] Kelsey S G, Kouznetsova Y, Makovetski B, et al. Batteries and battery systems: US, US7056617[P]. 2006.

[9] Zhang Y, Wang C Y, Tang X. Cycling degradation of an automotive LiFePO 4, lithium-ion battery[J]. Journal of Power Sources, 2011, 196(3):1513-1520.

[10] Weng C, Cui Y, Sun J, et al. On-board state of health monitoring of lithium-ion batteries using incremental capacity analysis with support vector regression [J]. Journal of Power Sources, 2013, 235(4):36-44.

[11] Nuhic A, Terzimehic T, Soczka-Guth T, et al. Health diagnosis and remaining useful life prognostics of lithium-ion batteries using data-driven methods $i \vec{z}[\mathrm{~J}]$. Journal of Power Sources, 2013, 239:680-688.

[12] Lin H T, Liang T J, Chen S M. Estimation of Battery State of Health Using Probabilistic Neural Network[J]. Industrial Informatics IEEE Transactions on, 2013, 9(2):679-685.

[13] Dong H, Jin X, Lou Y, et al. Lithium-ion battery state of health monitoring and 
remaining useful life prediction based on support vector regression-particle filter[J]. Journal of Power Sources, 2014, 271:114-123..

[14] Qin T, Zeng S, Guo J. Robust prognostics for state of health estimation of lithium-ion batteries based on an improved PSO-SVR model[J]. Microelectronics Reliability, 2015, 55(9):1280-1284.

[15] Sepasi S, Ghorbani R, Liaw B Y. Inline state of health estimation of lithium-ion batteries using state of charge calculation [J]. Journal of Power Sources, 2015, 299:246-254.

[16] Tong S, Klein M P, Park J W. On-line optimization of battery open circuit voltage for improved state-of-charge and state-of-health estimation [J]. Journal of Power Sources, 2015, 293:416-428.

[17] Liu D, Pang J, Zhou J, et al. Prognostics for state of health estimation of lithium-ion batteries based on combination Gaussian process functional regression[J]. Microelectronics Reliability, 2013, 53(6):832-839.

[18] Li F, Xu J. A new prognostics method for state of health estimation of lithium-ion batteries based on a mixture of Gaussian process models and particle filter [J]. Microelectronics Reliability, 2015, 55(7):1035-1045.

[19] Takeno K, Ichimura M, Takano K, et al. Quick testing of batteries in lithium-ion battery packs with impedance-measuring technology[J]. Journal of Power Sources, 2004, 128(1):67-75..

[20] Jungst R G, Nagasubramanian G, Case H L, et al. Accelerated calendar and pulse life analysis of lithium-ion cells[J]. Journal of Power Sources, 2003, s 119-121(6):870-873. .

[21] Prasad G K, Rahn C D. Model based identification of aging parameters in lithium ion batteries[J]. Journal of Power Sources, 2013, 232(232):79-85.

[22] Marcicki J, Canova M, Conlisk A T, et al. Design and parametrization analysis of a reduced-order electrochemical model of graphite/LiFePO 4, cells for $\mathrm{SOC} / \mathrm{SOH}$ estimation[J]. Journal of Power Sources, 2013, 237(3):310-324..

[23] Klass V, Behm M, Lindbergh G. Evaluating Real-Life Performance of Lithium-Ion Battery Packs in Electric Vehicles[J]. Journal of the Electrochemical Society, 2012, 159(11):A1856-A1560..

[24] Andre D, Appel C, Soczka-Guth T, et al. Advanced mathematical methods of SOC and SOH estimation for lithium-ion batteries[J]. Journal of Power Sources, 2013, 224(5):20-27.

[25] Tipireddy R, Nasrellah H A, Manohar C S. A Kalman filter based strategy for linear structural system identification based on multiple static and dynamic test data[J]. Probabilistic Engineering Mechanics, 2009, 24(1):60-74.

[26] Kim J, Cho B H. State-of-Charge Estimation and State-of-Health Prediction of a Li-Ion Degraded Battery Based on an EKF Combined With a Per-Unit System[J]. IEEE Transactions on Vehicular Technology, 2011, 60(9):4249-4260.

[27] Xiong R, Sun F, Chen Z, He H. A data-driven multi-scale extended Kalman filtering based parameter and state estimation approach of lithium-ion polymer battery in electric vehicles. Appl Energy 2014;113:463-76.

[28] Chen Z P, Wang Q T. The Application of UKF Algorithm for 18650-type Lithium Battery SOH Estimation [J]. Applied Mechanics \& Materials, 2014, 519-520:1077-1082..

[29] Haifeng D, Xuezhe W, Zechang S. A new SOH prediction concept for the power 
lithium-ion battery used on HEVs[C]// Vehicle Power and Propulsion Conference, 2009. VPPC '09. IEEE. 2009:1649-1653..

[30] Q, Wang, Y, Jiang, Y, Lu. State of Health Estimation for Lithium-ion Battery

Based on D-UKF[J]. International Journal of Hybrid Information Technology, 2015, 8(7): 55-70

[31] Han H, Ding Y S, Hao K R, et al. An evolutionary particle filter with the immune genetic algorithm for intelligent video target tracking[J]. Computers \& Mathematics with Applications, 2011, 62(7):2685-2695.

[32]Shafiei A, Momeni A, Williamson S S. Battery modeling approaches and management techniques for Plug-in Hybrid Electric Vehicles[C]// Vehicle Power and Propulsion Conference. IEEE, 2011:1-5.

[33] Zheng Y, Ouyang M, Lu L, Li J. Understanding aging mechanisms in lithium-ion battery packs: from cell capacity loss to pack capacity evolution [J]. J Power Sources 2015;278:287-95.

[34] Song Z, Hofmann H, Li J, et al. Optimization for a hybrid energy storage system in electric vehicles using dynamic programming approach [J]. Appl Energy 2015;139:151-62.

[35] Li J, Mazzola M S. Accurate battery pack modeling for automotive applications[J]. Journal of Power Sources, 2013, 237(237):215-228.

[36] Burgosmellado C, Orchard M E, Kazerani M, et al. Particle-filtering-based estimation of maximum available power state in Lithium-Ion batteries[J]. Applied Energy, 2016, 161.

[37] Park J, Dong J H, Park J S, et al. Hydrogen utilization as a fuel: hydrogen-blending effects in flame structure and $\mathrm{NO}$ emission behaviour of $\mathrm{CH} 4$-air flame[J]. International Journal of Energy Research, 2007, 31(5):472-485.

[38] Sanchez D M, Prado A F, Yokoyama T. On the effects of each term of the geopotential perturbation along the time I: Quasi-circular orbits[J]. Advances in Space Research, 2014, 54(6):1008-1018.

[39] Jun Bi, Wei Guan, LongTao Qi. A genetic resampling particle filter for freeway traffic-state estimation[J]. Chinese Physics B, 2012, 21(6):595-599.

[40] Barsali S, Ceraolo M. Dynamical models of lead-acid batteries: implementation issues[J]. IEEE Power Engineering Review, 2002, 22(2):63-63.

[41] Ivan T, Drábek Old?ich. Identification of nonlinear systems based on mathematical, physical analysis and least square method[J]. Acta Montanistica Slovaca, 2003, 8(4).

[42] Mojtaba S, Boroujeni S, Boroujeni B K, et al. Multi machine power system identification by using recursive least square method[J]. Indian Journal of Science \& Technology, 2011, 4(12).

[43] Wu Yu-ping. Lithium-ion battery application and practice[M].Beijing: Chemical industry press, 2004.

[44] Wen F, Jiang J, Zhang W A. Charging Method for Li-ion Battery Pack in Electric Vehicles[J]. Automotive Engineering, 2008 ,30:792-795.

[45] Jiang J C, Wen F, Wen J P, et al. Li-ion battery modeling and on-line model parameters identification for PEV[J]. Journal of Electric Power Science \& Technology, 2010,35:119-123.

[46] Niu L, Chen D, Guo H, et al. A Study on Predicting the Temperature Rise of a Lithium-ion Battery During Charging Based on ANFIS [J]. Automotive Engineering, 2014, 36(7):799-803.

[47] Haifeng D, Xuezhe W, Zechang S. A new SOH prediction concept for the power 
lithium-ion battery used on $\mathrm{HEVs}[\mathrm{C}] / /$ Vehicle Power and Propulsion Conference, 2009. VPPC '09. IEEE. 2009:1649-1653.

[48] Gui Chang-qing. Power battery[M]. Beijing: China Machine Press.

[49] Han H, Ding Y S, Hao K R, et al. An evolutionary particle filter with the immune genetic algorithm for intelligent video target tracking [J]. Computers \& Mathematics with Applications, 2011, 62(7):2685-2695.

[50] M.S. Arulampalam, S. Maskell, N. Gordon, T. Clapp, A tutorial on particle filters for online nonlinear/non-Gaussian Bayesian tracking, IEEE Transactions on Signal Processing 50 (2) (2002) 174-188 